\title{
PERCEPCIÓN DE LA SEXUALIDAD EN LA TITULACIÓN DE TRABAJO SOCIAL
}

\author{
LEYRE VAREA CORREA \\ GRADUADA EN TRABAJO SOCIAL \\ UNIVERSIDAD DE LA RIOJA \\ varea21@gmail.com
}

SUMARIO: I. INTRODUCCIÓN. II. MARCO TEÓRICO. III. METODOLOGÍA. IV. RESUlTAdOS Y DiSCUSIONES. V. RECOMENDACIONES. VI. BIBLIOGRAFÍA.

RESUMEN: La sexualidad es un valor humano que nace con la vida misma; Es el modo de vivirse, verse y sentirse como persona sexuada. No obstante, a pesar de considerarse un eje vertebrador de la vida de las personas, es un ámbito que no se está abordando de manera integral ni desde los centros educativos ni desde los hogares, provocando una distorsión de la información sobre sexualidad en la población joven. En este estudio se contextualizan las competencias de una muestra de 112 estudiantes de la titulación de Trabajo Social sobre la Educación Sexual Integral.

PALABRAS CLAVE: Sexualidad, educación sexual, modelo integrador, Trabajo Social, centro educativo.

\section{PERCEPTION OF SEXUALITY IN SOCIAL WORK DEgREE}

ABSTRACT: Sexuality is a human value that is born with life itself; It is the way to live, look and feel like a sexed person. However, despite being considered a backbone of people's lives, it is an area that is not being comprehensively addressed either from schools or from homes, causing a distortion of information on sexuality in the young population. This study contextualizes the competencies of a sample of 112 students of the Social Work degree on Comprehensive Sex Education.

KEYWORDS: Sexuality, sex education, integrative model, Social Work, school. 


\section{Introducción}

Según la Organización Mundial de la Salud, la sexualidad es un ingrediente básico en la vida de las personas desde su nacimiento. Se expresa a través de los pensamientos, fantasías, deseos, creencias, actitudes, valores, comportamientos, prácticas, roles y relaciones.

La sexualidad está influenciada por la interacción de factores biológicos, psicológicos, sociales, económicos, políticos, culturales, éticos, legales, históricos, religiosos y espirituales, e involucra a todas las personas ${ }^{1}$. Por lo tanto, no es un fenómeno exclusivamente biológico ni un hecho exclusivamente individual.

Cada ser humano vive y experimenta su sexualidad de diversas formas, hacia múltiples fines y en todas las etapas de su vida. Esto le posibilita conocerse a sí mismo, conocer a las demás personas y conocer las relaciones interpersonales en un determinado contexto social2.

Sin embargo, durante muchos años, la sociedad ha identificado la sexualidad únicamente con la reproducción, condenando, entre otras cosas, la diversidad o los actos sexuales que pudieran transcurrir fuera del matrimonio.

Este hecho ha provocado que hoy en día coexistan múltiples confusiones y tabúes sexuales por parte de las familias y de la escuela -donde sólo se abordan las infecciones de transmisión sexual y el embarazo no deseado en la adolescencia-, lo que fomenta que la juventud obtenga la información de otras fuentes -Internet y amistades-.

En este sentido, se evidencia la necesidad legislativa de regular una Educación Sexual Integral (en adelante ESI) que se imparta en todos los centros educativos españoles desde el primer ciclo de la vida -Educación Infantil-.

Ahora bien, la responsabilidad de educar en sexualidad no es exclusiva de los centros educativos, sino que debe ser compartida con familiares y profesionales de otros ámbitos como, por ejemplo, del Trabajo social.

\section{Marco teórico}

La Cumbre de las Naciones Unidas fijó en el año 2015 unas metas que componen la Nueva Agenda 2030 y que giran en torno a cinco ejes centrales: Planeta, personas, prosperidad, paz y alianzas. Dichas metas, formalmente denominadas "Objetivos de Desarrollo Sostenible" (en adelante ODS), cumplen una labor de trascendencia universal para poder lograr un mundo más justo. Los ODS que articulan este trabajo son los siguientes 3 :

- ODS no 3: "Salud y bienestar".

- ODS no 4: "Educación de calidad".

\footnotetext{
1 World Health Organization, Defining Sexual Health. Report of a technical consultation on sexual health, 2006 (Disponible en: https://www.who.int/reproductivehealth/publications/sexual_health/defining_sh/en/; fecha de consulta 20/10/2018)

2 Padrón, M.M.; Fernández, L.; Infante, A.; París, A., El Libro Blanco sobre Educación Sexual de la Provincia de Málaga, Málaga, Diputación de Málaga, Área de Juventud y Deportes, 2007.

3 Naciones Unidas, Objetivos de Desarrollo Sostenible, 2015 (Disponible en: https://www.un.org/sustainabledevelopment/es/objetivos-de-desarrollo-sostenible/; fecha de consulta 02/11/2018).
} 
- ODS no 5: "Igualdad de género".

Estos objetivos deben abordarse desde el modelo integrador, según el cual se imparte una ESI global. Sin embargo, en el sistema educativo y comunitario conviven otros dos modelos de educación sexual que evidencian trasfondos ideológicos: El modelo tradicional, que asemeja la educación sexual a la educación moral y condena el placer y los anticonceptivos, y el modelo preventivo, que considera la educación sexual únicamente como un medio para evitar los riesgos asociados al sexo -entendiendo el sexo como la actividad sexual-4.

Descartando los dos últimos modelos, un enfoque basado en los derechos del modelo integrador busca dotar a las personas con los conocimientos, habilidades, actitudes y valores necesarios para definir y gozar de su sexualidad de manera individual, física y emocional. Este aprendizaje se logra abordando todos los componentes que articulan la ESI5:

- Sexo: Hace referencia a las características biológicas que clasifican a las personas como hombres, mujeres o intersexuales.

- Género: Concepto que añade la dimensión social para determinar lo que implica "ser hombre" y "ser mujer" en una sociedad.

- Identidad de género: Se relaciona con la percepción que construye cada persona sobre sí misma, reconociéndose como hombre, mujer o ninguno de los géneros binarios.

- Expresión de género: Forma en la que se exterioriza la identidad de género a través del lenguaje, la apariencia, etc.

- Diversidad y orientación sexual: Se determina según el género de la persona por la que se siente atracción física, romántica o sexual.

- Salud sexual y reproductiva y VIH: Se abordan las infecciones de transmisión sexual, el ciclo de vida, etc.

- Ciudadanía y derechos sexuales: Se incluyen los derechos humanos internacionales, las políticas nacionales y las barreras sociales, culturales y éticas.

- Placer: Se reconoce el placer de la sexualidad en todas las etapas de la vida.

- Violencia: Se identifican los tipos de violencia y el sexo no consensual.

- Tipos de relaciones y familias y emociones e intimidad.

Pero ¿cuándo debe comenzar dicho aprendizaje? Siguiendo a Luz de Santiago (2009) las áreas de actuación de la ESI son las siguientes ${ }^{6}$ :

\footnotetext{
${ }^{4}$ Padrón, M.M. et. al., El Libro Blanco..., op. cit.

${ }^{5}$ International Planned Parenthood Federation., IPPF Framework for Comprehensive Sexuality Education, Londres, International Planned Parenthood Federation (IPPF), 2010.

${ }^{6}$ Santiago, L., Educación sexual, Madrid, Ministerio de Educación, Gobierno de España, 2009 (Disponible en: https://leer.amazon.es/?asin=B01AAB3DDY; fecha de consulta 09/05/2019).
} 
- ESI en infantil: Se debe promover un desarrollo responsable, saludable, autónomo y asertivo de las niñas y los niños.

- ESI en primaria: Se deben prevenir las dudas, inseguridades y miedos que acompañan la pubertad.

- ESI en secundaria: Se debe prevenir y promover una sexualidad responsable, cuestionando la información procedente de Internet, la publicidad, etc.

- ESI en ciclos superiores: Se debe enseñar a abordar este tema desde cualquier disciplina y con cualquier persona.

- ESI para familias: Se debe destacar la importancia del diálogo y la confianza para gestionar la sexualidad responsable de las hijas y los hijos.

- ESI para el público en general: Se debe tratar el conocimiento del propio cuerpo en las diferentes etapas de la vida.

En definitiva, la educación en sexualidad empodera a niñas, niños, adolescentes, jóvenes y personas adultas para que tomen sus propias decisiones respetando los Derechos Humanos, fomentando la igualdad de género y considerando, desde una perspectiva positiva, la diversidad, las relaciones, la reproducción, el placer y la prevención de las infecciones de transmisión sexual. Todo ello partiendo del respeto, la empatía, la responsabilidad y la inclusión.

Esta visión integradora de la educación sexual debe verse reflejada en el plan de estudios de los centros educativos españoles. Sin embargo, España carece de una obligación legislativa que verse sobre la inclusión de la temática sexual en el currículum escolar ${ }^{7}$, trasladando la responsabilidad a los diferentes centros y a profesionales de otras disciplinas.

En el caso del Trabajo Social, se necesita una redefinición de la profesión que le permita construir una mirada adaptada a la realidad social con la que convive, sin limitar su discurso únicamente a los grupos minoritarios (colectivo LGTBI) ${ }^{8}$.

En este sentido, el modelo PLISSIT ${ }^{9}$ describe cuatro secciones orientadas a satisfacer las necesidades de las personas usuarias:

- Dar "Permiso" (Permission, P) para hablar de sexualidad, creando un clima confortable y seguro, respetando la intimidad y evitando prejuicios.

\footnotetext{
${ }^{7}$ La Ley Orgánica 8/2013, de 9 de diciembre, para la Mejora de la Calidad Educativa, suprimió la "Educación para la Ciudadanía y los Derechos Humanos"; única asignatura que incluía contenidos sobre sexualidad.

${ }^{8}$ Langarita, J. A.; Mesquida, J.M., "Interpelaciones de la sexualidad (es) al trabajo social. Apuntes para una praxis renovada", en Raya, E.; Pastor, E., Trabajo Social, Derechos Humanos e Innovación Social, Navarra, Aranzadi, 2016, págs. 171-182.

9 Según Ayaz (2013) y Malón (2009) el modelo PLISSIT fue creado con la finalidad de ayudar a profesionales de la salud a abordar temas relacionados con la sexualidad y, posteriormente, se aplicó al Trabajo social.

Ayaz, S., "Sexuality and Nursing Process: A literature Review", Sexuality and disability, 31, 2013, págs. 17-30, (Disponible en https://www.researchgate.net/publication/257663534_Sexuality_and_Nursing_Process_A_ Literature_Review; fecha de consulta: 15/11/2019)

Malón, A., Niveles de intervención profesional: el modelo PLISSIT, Sexualidad. Planteamientos y claves para la intervención profesional en el ámbito de la discapacidad, Huesca, CADIS, 2009, págs. 101-107.
} 
- Dar "Información Limitada" (Limited Information, LI) a las personas usuarias para que cuenten los detalles, desmitificando las creencias erróneas y facilitando la información concreta.

A través de estas dos fases, se obtiene la historia previa.

- Dar "Sugerencias Específicas" (Specific Suggestions, SS) para intentar solucionar el o los problemas específicos de la persona.

- Si estas pautas de intervención no son suficientes, se deriva a la persona a una/un especialista que le programe "Terapia Intensiva" (Intensive Therapy, IT) de largo plazo.

Con este modelo se pretende que las personas profesionales de Trabajo Social sean consideradas un punto de referencia al que acudir ante cualquier experiencia, crisis o problema vinculados a la sexualidad.

\section{Metodología}

Con motivo de continuar la línea del objetivo general: "Indagar en torno a la necesidad de impartir la ESI en todos los centros educativos a partir de la propia experiencia en sexualidad del alumnado de Trabajo Social de la Universidad de La Rioja", se ha realizado una revisión bibliográfica y se ha desarrollado una metodología de carácter cuantitativo. De esta forma, los resultados obtenidos se enfocan hacia una explicación causal de los hechos sociales.

La técnica de investigación a emplear en la metodología cuantitativa es la encuesta, con el fin de obtener gran cantidad de información en un breve periodo de tiempo. Además, esta técnica permite agrupar y comparar las respuestas sobre la formación y la percepción del alumnado acerca de la sexualidad y sus implicaciones.

Su instrumento de recogida de información es el cuestionario; un cuestionario de carácter personal y anónimo que ha sido diseñado tomando dos fuentes de referencia. La primera fuente es el Centro de Investigaciones Sociológicas y la segunda son las "X Jornadas sobre Diversidad AfectivoSexual y de Género: Intersexualidad" desarrolladas en la Universidad de La Rioja en el año 2019.

Cada fuente moldea una parte del cuestionario. La primera parte está formada por nueve preguntas agrupadas en tres bloques: "Fuentes de información reales y deseadas sobre sexualidad", "Educación sexual en los centros educativos" y "Conocimientos conceptuales sobre sexualidad". La segunda parte trata del visionado de un vídeo en el que salen dos personas -María y Adamconversando en la cocina sobre cómo organizarse para acudir a una cena, pero, una de ellas, no se muestra conforme con la relación que les une. Una vez visto, se les pregunta sobre el sexo, género, orientación sexual, etc. de ambas personas. Esta parte configura el cuarto bloque del cuestionario: "Conocimientos conceptuales aplicados a la práctica".

Para la realización del estudio se ha seleccionado una muestra de conveniencia y no probabilística. Dicha muestra objeto de estudio se extrajo de la titulación de Trabajo Social de la Universidad de la Rioja. Más concretamente, aunque en el grado hay matriculadas/os un total de 170 estudiantes, el cuestionario lo han realizado $112-22$ hombres y 90 mujeres-, cuyas edades oscilan entre los 18 y los 27 años, siendo 21 la edad más frecuente. 


\section{Resultados y discusiones}

La forma en la que las personas viven y expresan su sexualidad influye en la forma en la que configuran su personalidad y su autoaceptación, y viceversa. Sin embargo, la sexualidad sigue siendo hoy en día un tema tabú y, por lo tanto, mitificado. Son múltiples los estudios ${ }^{10}$-incluido este trabajo- que demuestran que las temáticas sexuales que se están abordando en los centros educativos no son suficientes. Este hecho provoca que la juventud sea uno de los colectivos más vulnerables a abusos, explotaciones, embarazos e infecciones.

A la problemática de los colegios, donde solo el 49\% del grupo encuestado recibió alguna clase o taller sobre educación sexual durante su infancia, se suma otro ámbito donde tampoco se aborda la sexualidad; el familiar. La mayor parte del grupo comparte sus experiencias sexuales solo con sus amistades (94\%) y soluciona sus dudas a través de Internet y otros medios de comunicación (88\%). Por ello, el $86 \%$ de las personas reclaman una educación transversal en la que participen, de manera conjunta, familiares, profesorado y otras/os profesionales.

Esta educación -que no enseñanza ${ }^{11}$ - no debe tratar solo el embarazo no deseado y las infecciones de transmisión sexual, tal y como se viene haciendo hasta ahora, sino que debe mostrar una visión más integradora. Un elevado porcentaje del grupo encuestado considera que también deben abordarse la violencia y los abusos sexuales (90\%); las relaciones de pareja, las diversas concepciones de sexualidad, las maneras de obtener y dar placer y la diversidad sexual (88\%); y la reproducción y anatomía genital, los modelos de familia y las prácticas sexuales $(82 \%)$. Aunque la pornografía y la prostitución obtuvieron porcentajes inferiores, no dejan de ser un tipo de violencia y abuso.

Ante tal situación, es importante recordar que la Educación Sexual Integral no puede vincularse únicamente a un programa educativo, a una institución concreta o a un único curso, sino a un trabajo colaborativo y permanente en el tiempo en el que toda la sociedad se vea inmersa; incluidos la familia y el personal profesional de Trabajo Social. Pero para que esto sea posible, hay que dominar determinados conocimientos sobre la sexualidad ${ }^{12}$.

Sin embargo, un 70\% del alumnado de Trabajo Social confunde los términos asociados a la sexualidad -exceptuando los referidos a la orientación sexual- tanto a la hora de definirlos como a la hora de ponerlos en práctica, desligándose de la línea de definiciones de la Organización Mundial de la Salud.

Además, a través del caso práctico, un $86 \%$ presupone el sexo -hembra y macho- de María y Adam y un $74 \%$ su género - mujer y hombre-, a pesar de ser aspectos que no pueden conocerse a simple vista. Respecto a la expresión de género, el 87\% sí que acierta al afirmar que la de María es

\footnotetext{
${ }^{10}$ Se pueden consultar los artículos "Orientaciones técnicas internacionales sobre educación en sexualidad. Un enfoque basado en la evidencia” de la UNESCO y "Coeducación: dos sexos en un solo mundo" del Ministerio de Educación, Cultura y Deporte.

${ }^{11}$ Mientras enseñar es transmitir conocimientos ya programados, educar es transmitir determinados valores que estimulen el espíritu crítico y la defensa de derechos.

${ }^{12}$ Cabe recordar que no se trata de que las/os profesionales de Trabajo social sean expertas/os en la temática sexual, si no que se consideren un punto de referencia en los casos en que se necesite asesoramiento.
} 
femenina y la de Adam masculina. Dentro de la orientación sexual, afirman la heterosexualidad de ambas personas, yendo más allá al afirmar que son pareja $(85 \%)^{13}$.

Esto es probablemente un reflejo de que lo aceptado socialmente es la heterosexualidad y el binarismo, por lo que se debe hacer un mayor esfuerzo por visibilizar todo aquello que no cumpla con tal ideal. Ni todas las personas son heterosexuales ni tienen por qué reconocerse como mujeres o como hombres ${ }^{14}$.

Por último, un gran porcentaje del alumnado de Trabajo Social afirma sentirse preparado para abordar cualquier temática con las personas usuarias $(66 \%)$. Ahora bien, en caso de tener alguna duda, solo una pequeña parte del grupo sabe a dónde podría acudir (29\%); el mismo porcentaje que no conoce ninguna entidad que trabaje, de manera exclusiva, la sexualidad.

\section{Recomendaciones}

Se propone continuar esta investigación con una síntesis de buenas prácticas en Educación Sexual Integral en España. Se consideran igual de válidos los casos en los que se eduquen a estudiantes como aquellos en los que se formen a familiares y profesionales que muestren interés en la temática.

Además de la investigación propuesta, se sugieren posibles líneas de intervención futura desde la disciplina de Trabajo Social:

- Incidir en el salto del modelo tradicional y del modelo preventivo al modelo integrador de educación sexual.

- Evitar la difusión de mitos infundados sobre los efectos de educar en sexualidad a partir de la Educación Infantil.

- Fomentar las intervenciones en las que no se prejuzgue ni encasille a las personas usuarias.

- Destacar el papel de la persona profesional de Trabajo Social como agente de información, apoyo y asesoramiento sexual.

\section{Bibliografía}

AYAZ, S., "Sexuality and Nursing Process: A literature Review", Sexuality and disability, 31, 2013, pp. 17-30, (Disponible en https://www.researchgate.net/publication/257663534_Sexuality _and_Nursing_Process_A_Literature_Review; fecha de consulta: 15/11/2019).

INTERNATIONAL PLANNED PARENTHOOD FEDERATION., IPPF Framework for Comprehensive Sexuality Education, Londres, International Planned Parenthood Federation (IPPF), 2010.

\footnotetext{
${ }^{13} \mathrm{El}$ vídeo es tan breve que la única información que se puede extraer del mismo es la referida a la expresión de género; en ningún momento las personas hablan sobre la relación que les une; sobre su sexo asignado; o sobre el género con el que se identifican.

${ }^{14}$ Langarita, J. A.; Mesquida, J.M. "Interpelaciones..., op. cit.
} 
LANGARITA, J. A.; MESQUIDA, J. M., "Interpelaciones de la sexualidad (es) al trabajo social. Apuntes para una praxis renovada”, en E. Raya y E. Pastor, Trabajo Social, Derecbos Humanos e Innovación Social, Navarra, Aranzadi, 2016, pp. 171-182.

MALÓN, A., Niveles de intervención profesional: el modelo PLISSIT, Sexualidad. Planteamientos y claves para la intervención profesional en el ámbito de la discapacidad, Huesca, CADIS, 2009, pp. 101-107.

NACIONES UNIDAS, Objetivos de Desarrollo Sostenible, 2015 (Disponible en: https://www.un.org/sustainabledevelopment/es/objetivos-de-desarrollo-sostenible/; fecha de consulta 02.11.2018).

PADRÓN, M. M.; FERNÁNDEZ, L.; INFANTE, A.; PARÍS, A., El Libro Blanco sobre Educación Sexual de la Provincia de Málaga, Málaga, Diputación de Málaga, Área de Juventud y Deportes, 2007.

SANTIAGO, L., Educación sexual, Madrid, Ministerio de Educación, Gobierno de España, 2009, (Disponible en: https://leer.amazon.es/?asin=B01AAB3DDY; fecha de consulta 09/05/2019).

WORLD HEALTH ORGANIZATION, Defining Sexual Health. Report of a technical consultation on sexual health, 2006 (Disponible en: https:/ /www.who.int/reproductivehealth/publications/sexual_ health/defining_sh/en/; fecha de consulta 20/10/2018). 